\title{
Probing the evolution of molecular cloud structure
}

\section{From chaos to confinement}

\author{
J. Kainulainen ${ }^{1}$, H. Beuther ${ }^{1}$, R. Banerjee ${ }^{2}$, C. Federrath ${ }^{2,3}$, and T. Henning ${ }^{1}$ \\ 1 Max-Planck-Institute for Astronomy, Königstuhl 17, 69117 Heidelberg, Germany \\ e-mail: [jtkainul; beuther; henning] @mpia . de \\ 2 Zentrum für Astronomie der Universitat Heidelberg, Institut für Theoretische Astrophysik, 69120 Heidelberg, Germany \\ ${ }^{3}$ Ecole Normale Supérieure de Lyon, CRAL, 69364 Lyon, France
}

Received 21 December 2010 / Accepted 27 March 2011

\section{ABSTRACT}

\begin{abstract}
We present an analysis of the large-scale molecular cloud structure and of the stability of clumpy structures in nearby molecular clouds. In our recent work, we identified a structural transition in molecular clouds by studying the probability distributions of their gas column densities. In this paper, we further examine the nature of this transition. The transition takes place at the visual extinction of $A_{\mathrm{V}}^{\text {tail }}=2-4 \mathrm{mag}$, or equivalently, at $\Sigma^{\text {tail }} \approx 40-80 M_{\odot} \mathrm{pc}^{-2}$. The clumps identified above this limit have wide ranges of masses and sizes, but a remarkably constant mean volume density of $\bar{n} \approx 10^{3} \mathrm{~cm}^{-3}$. This is $5-10$ times higher than the density of the medium surrounding the clumps. By examining the stability of the clumps, we show that they are gravitationally unbound entities, and that the external pressure from the parental molecular cloud is a significant source of confining pressure for them. Then, the structural transition at $A_{\mathrm{V}}^{\text {tail }}$ may be linked to a transition between this population and the surrounding medium. The star-formation rates in the clouds correlate strongly with the total mass in the clumps, i.e., with the mass above $A_{\mathrm{V}}^{\text {tail }}$, and drops abruptly below that threshold. These results imply that the formation of pressure-confined clumps introduces a prerequisite for star formation. Furthermore, they give a physically motivated explanation for the recently reported relation between the star-formation rates and the amount of dense material in molecular clouds. Likewise, they give rise to a natural threshold for star formation at $A_{\mathrm{V}}^{\text {tail }}$.
\end{abstract}

Key words. ISM: clouds - ISM: structure - stars: formation - dust, extinction - evolution

\section{Introduction}

Formation of dense, self-gravitating structures inside more diffuse, large-scale molecular clouds is the ultimate prerequisite for star formation. In addition to self-gravitating dense cores, molecular clouds in which star formation is taking place show exhaustive structural complexity characterized by large contrasts in both density and velocity. From the general observation that almost all known molecular clouds harbor young stars, it is known that the formation of structures capable of star formation (or alternatively, cloud dissipation) must proceed relatively rapidly compared to the life-times of molecular clouds. Likely as a result of this complexity and rapid development, molecular clouds also show wide ranges of star-forming efficiencies and -rates (e.g. Heiderman et al. 2010; Lada et al. 2010). This connection between the cloud structure and the capability of a cloud to form stars makes determining the roles of processes and parameters that control the cloud structure a fundamental open topic in the physics of star formation (reviewed, e.g., by McKee \& Ostriker 2007; Mac Low \& Klessen 2004).

In the current analytic models of star formation, one particularly important structural parameter of molecular clouds is the probability density function (PDF, hereafter) of volume densities, which describes the probability of a volume $\mathrm{d} V$ to have a density between $[\rho, \rho+\mathrm{d} \rho]$. In these theories, the function has pivotal role: it is used to explain among others the initial mass function of stars and the star formation rates and efficiencies of molecular clouds (e.g. Padoan \& Nordlund 2002; Krumholz \& McKee 2005; Elmegreen 2008; Hennebelle \& Chabrier 2009). In particular, this distribution is expected to take a log-normal shape in isothermal, turbulent media that are not significantly affected by the self-gravity of gas (e.g. Vázquez-Semadeni 1994; Padoan et al. 1997; Ostriker et al. 1999; Federrath et al. 2008b). Most importantly from the observational point-of-view, the lognormality of the distribution is expected to be reflected in the probability distributions of column densities in molecular clouds (Vázquez-Semadeni \& García 2001; Goodman et al. 2009; Federrath et al. 2010). Also, a method has recently been developed to attain information of the actual three-dimensional density PDF based on the observed, two-dimensional column density PDFs (Brunt et al. 2010a,b; Brunt 2010). Even though it has been pointed out that the general log-normal-like form for the (column) density PDF can be borne out by various processes (Tassis et al. 2010), it is obvious that a reliable theory of cloud structure must agree with the observed characteristics of the distribution. This is particularly the case if the probability distribution shows any scale-dependent features and/or time evolution. These properties have indeed been predicted, e.g. in the presence of strong self-gravity (Klessen 2000; Federrath et al. 2008a; Cho \& Kim 2011; Kritsuk et al. 2011), and scale-dependent features have also recently been observed (Kainulainen et al. 2009b; Froebrich \& Rowles 2010; Pineda et al. 2010b). This makes probing the column density probability distributions one parametner of cloud structure that can be used to constrain analytic star formation theories.

However, the connection between theoretical and numerical predictions with observations of the PDF has scarcely been investigated. The studies in which the column density PDFs of mostly individual clouds have been examined have found a 
qualitative agreement with the predicted log-normal shape (e.g., Ridge et al. 2006b; Goodman et al. 2009; Butler \& Tan 2009). The lack of systematic studies of column density PDFs has been mostly due to observational obstacles: all observational tracers of the cloud mass distribution suffer from shortcomings specific to the tracer in question (see, e.g., Goodman et al. 2009). Generally, the dynamical ranges probed by different molecular emission line tracers are often narrow, thereby probing only a limited range of the PDF. Dust continuum emission observations probe a wider dynamical range of column densities, but become insensitive at column densities below $N \lesssim$ a few $\times 10^{21} \mathrm{~cm}^{-2}$, thus missing a regime where most of the cloud mass is. This is the case also for dust extinction measurements using infrared shadowing features. In addition to these restrictions, mapping nearby cloud complexes that often span several degrees on the sky at high sensitivity requires a colossal observational effort, which is not generally feasible through typical observing campaigns. Dust extinction mapping in the near-infrared reaches only modest column densities of $N \lesssim$ a few $\times 10^{22} \mathrm{~cm}^{-2}$, thereby mostly missing dense star-forming clumps and cores. However, near-infrared extinction mapping reaches the low column densities $N \sim 1-3 \times 10^{22} \mathrm{~cm}^{-2}$ very efficiently (Lombardi \& Alves 2001), a regime where most of the cloud mass is. Therefore, it provides a feasible tool to measure the column density PDFs at the scales of entire cloud complexes. Near-infrared extinction mapping has indeed been used for this purpose recently, especially by Kainulainen et al. (2009b; see also Lombardi et al. 2006, 2008b; Froebrich \& Rowles 2010; Lombardi et al. 2010; Pineda et al. 2010b).

In our recent work (Kainulainen et al. 2009b, Paper I hereafter), we presented the first systematic study of the column density PDFs in all nearby molecular clouds closer than 200 pc. We used near-infrared dust extinction maps of 23 molecular clouds to identify a transition in the PDF shape from a log-normal-like shape at lower column densities to a power-law-like shape at higher column densities. This transition is characteristic to all star-forming molecular clouds. However, we showed that some of the non-star-forming clouds in our sample did not have the transition, but their PDFs were well fitted by a log-normal over the entire range of column densities above the detection limit. This led us to speculate that the PDF feature is linked to a transition from a quiescent regime dominated by turbulent motions to a regime of active star formation dominated by gravity. The non-star-forming clouds that do not show the feature would then entirely belong to the former regime, and during their subsequent evolution toward star formation, gravitationally dominated structures would appear that would also induce a transition of the shape of their PDFs.

In this paper, we present a more detailed analysis of the structural transition that is identified from the column density PDFs. In particular, we will examine the physical characteristics and stability of the structures that are identified with the PDFs. With this analysis, we will show that the change in the PDF shape can be understood as a transition between the diffuse, interclump medium and a population of clumps that are gravitationally unbound, but are significantly supported against dispersal by the external pressure imposed on them by the surrounding medium. This interpretation links the PDF shape to a physically motivated explanation for the relation between star-formation rates and the amount of high-density material in molecular clouds that was recently reported by Lada et al. (2010), and it also connects it to the threshold of star formation in molecular clouds.

In Sect. 2 we briefly describe the dust column density data used in this paper. In Sect. 3 we characterize the structures identified from the column density maps and examine their stability. In Sect. 4 we discuss the results and their impact for the structure- and star formation in molecular clouds. In Sect. 5 we give our conclusions.

\section{The column density data of nearby clouds}

In Paper I we used the near-infrared color-excess mapping method presented by Lombardi (2009; see also Lombardi 2005; Lombardi \& Alves 2001), called nicest, to derive dust extinction maps for 23 nearby molecular clouds. The technique was used in conjunction with near-infrared data from the 2MASS survey (Skrutskie et al. 2006), resulting in dust column density maps covering the dynamical range of $A_{\mathrm{V}} \approx 1.2-25 \mathrm{mag}$ in the spatial resolution of $0.1 \mathrm{pc}\left(\sim 2^{\prime}\right.$ at the distance of the nearby clouds). While the cloud sample for this paper is otherwise the same as in Paper I, we excluded the Coalsack cloud from the analysis. Our recent molecular line observations of the Coalsack have shown that the region likely includes a significant extinction component not only from Coalsack, but also from an extended cloud at a larger distance (Beuther et al., in prep.). Since the effect of that component may well disturb the statistics derived in this paper, we decided to exclude Coalsack from the sample.

As an example of our data, Fig. 1 shows the extinction map derived for the Ophiuchus cloud. The figure also shows the PDF of the cloud, with the lognormal-like low- $A_{\mathrm{V}}$ part and the powerlaw-like tail at high- $A_{\mathrm{V}}$ clearly separate. The transition between these parts occurs approximately at $A_{\mathrm{V}}^{\text {tail }} \approx 2.8 \mathrm{mag}$ in this cloud. Throughout this paper, we refer to this position in the PDFs as $A_{\mathrm{V}}^{\text {tail }}$. In the clouds included in the study, the transition occurs at relatively low $A_{\mathrm{V}}$ values, $A_{\mathrm{V}}^{\text {tail }}=[2.0,11] \mathrm{mag}$, although in most cases between $A_{\mathrm{V}}^{\text {tail }}=2-4$ mag. The $A_{\mathrm{V}}^{\text {tail }}$ values determined for each cloud are listed in Table 1.

The $A_{\mathrm{V}}^{\text {tail }}$ value defines a set of spatially closed iso-contours in the column density maps (see Fig. 1). Throughout the paper, we will refer to the region where $A_{\mathrm{V}} \lesssim A_{\mathrm{V}}^{\text {tail }}$ as the diffuse component, and similarly, to all regions where $A_{\mathrm{V}} \gtrsim A_{\mathrm{V}}^{\text {tail }}$ as the dense component. The former refers then by definition to the lognormal part of the PDF and the latter to the power-law-like part. Morphologically, the diffuse component is a uni-body structure in all complexes, but the dense component forms separate structures. We will refer to these separate structures as clumps in this paper.

\section{Results}

In this section, we use the column density data introduced in Sect. 2 to examine the nature of the diffuse and dense components. We first examine the physical characteristics of the components, namely the masses and sizes, densities, and velocity structure. Then, we consider the observed characteristics from the point of view of pressure balance in molecular clouds.

\subsection{Characteristics of the diffuse and dense components}

\subsubsection{Total mass}

The gas column densities in the clouds can be inferred from the extinction maps using the measured extinction-to-gas column ratio. We transformed the visual extinction values in the maps into hydrogen column densities using the relation (Bohlin et al. 1978)

$\beta=\left(N(\mathrm{H})+2 N\left(\mathrm{H}_{2}\right)\right) / A_{\mathrm{V}}=1.9 \times 10^{21} \mathrm{~cm}^{-2} \mathrm{mag}^{-1}$. 


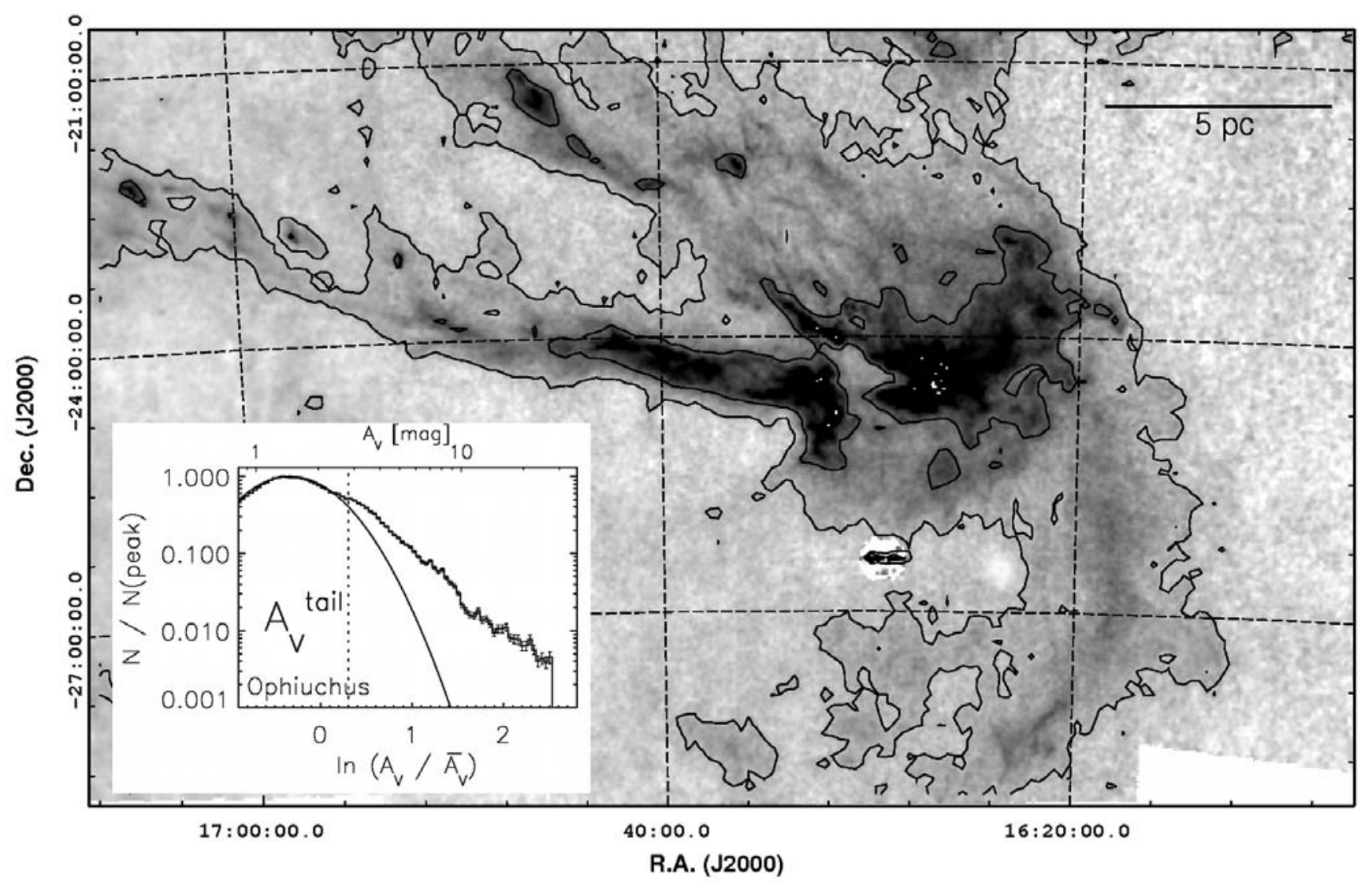

Fig. 1. Dust extinction through the Ophiuchus molecular cloud, derived using the near-infrared extinction mapping technique and $2 \mathrm{MASS}$ data (Paper I). The contours are drawn at $A_{\mathrm{V}}^{\text {tail }}=2.8 \mathrm{mag}$, and at $A_{\mathrm{V}}=1$ mag which is used to calculate the total mass of the cloud. The mass in the dense component is $\sim 21 \%$ of the mass above $A_{\mathrm{V}}=1 \mathrm{mag}$ in this cloud (cf. Table 1 ). The inset shows the column density PDF of the cloud, with an approximate log-normal function fitted to the peak of it.

We then calculated the total mass of the cloud as a sum of extinction values above $A_{\mathrm{V}}=1 \mathrm{mag}$

$M_{\mathrm{tot}}=D^{2} \mu \beta \times \int_{\Omega: A_{\mathrm{V}}>1 \mathrm{mag}} A_{\mathrm{V}} \mathrm{d} x \mathrm{~d} y$,

where $D$ is the distance to the cloud, $\mu=1.37$ is the mean molecular weight (adopting the same values as Lombardi et al. 2008 b, i.e., $63 \%$ hydrogen, $36 \%$ helium, and $1 \%$ dust), and $x$ and $y$ refer to the map pixels. We adopt the same distances for clouds as listed in Paper I. We note that the chosen lower limit of $A_{\mathrm{V}}=1 \mathrm{mag}$ is arbitrary, and the total mass depends on the selected value (choosing a lower threshold will yield higher masses for all clouds). However, a fixed value will make the values comparable between the clouds. We also note that the $A_{\mathrm{V}}=1 \mathrm{mag}$ contour is closed in most mapped regions, which therefore uniformly defines a cloud boundary. Table 1 lists the mean extinctions, $\bar{A}_{\mathrm{V}}$, for the clouds calculated with this definition for a cloud.

The total mass of the dense component was calculated from the extinction in excess to the $A_{\mathrm{V}}^{\text {tail }}$ threshold level:

$M_{\text {dense }}=D^{2} \mu \beta \times\left[\int_{\Omega: A_{\mathrm{V}}>A_{\mathrm{V}}^{\text {tail }}} A_{\mathrm{V}} \mathrm{d} x \mathrm{~d} y-A_{\mathrm{V}}^{\mathrm{tail}} \times \int_{\Omega: A_{\mathrm{V}}>A_{\mathrm{V}}^{\mathrm{tai}}} \mathrm{d} x \mathrm{~d} y\right]$.

The mass of the diffuse component was then defined as $M_{\text {diffuse }}=$ $M_{\mathrm{tot}}-M_{\mathrm{dense}}$. We list in Table 1 the ratios of the dense component mass to the total mass of the cloud. Clearly, the mass of the diffuse component dominates the cloud mass in all clouds. The ratios vary from a few percents for clouds with low star-forming activity to $\sim 20 \%$ for the most active clouds. Note that the total mass of the cloud was calculated as the mass above $A_{\mathrm{V}}>1$ mag. Because the column density below this level is, of course, not zero, our total masses represent lower limits. Accordingly, the quoted $M_{\text {dense }} / M_{\text {tot }}$ ratios represent upper limits.

\subsubsection{Clumps in the dense component}

In the following, we characterize the individual structures, i.e. clumps, in the dense component. We used a simple thresholding approach for identifying the clumps from the extinction maps, namely the "clumpfind2d" routine (Williams et al. 1994). All pixels in the map that are connected with each other and above $A_{\mathrm{V}}^{\text {tail }}$ are considered as one clump. We emphasize that we made no effort to identify single-peaked structures nested inside the contour defined by $A_{\mathrm{V}}^{\text {tail }}$, because we particularly aimed to examine the mean physical parameters inside regions defined by the $A_{\mathrm{V}}^{\text {tail }}$ threshold. Therefore, there can be numerous distinct column density peaks (of any column density higher than $A_{\mathrm{V}}^{\text {tail }}$ ) nested inside the clumps. In terms of the clumpfind $2 \mathrm{~d}$ algorithm, this approach equals using $A_{\mathrm{V}}^{\text {tail }}$ as a threshold level for structure detection, but not defining any additional column density levels that would be used in detecting peaks inside this parental structure.

This exercise identified about 10 clumps from each cloud complex that show wide ranges of sizes and masses. The effective radii of the clumps, defined as $R=\sqrt{A / \pi}$ where $A$ is 
Table 1. Molecular clouds and the derived properties.

\begin{tabular}{|c|c|c|c|c|c|c|}
\hline Cloud & $A_{\mathrm{V}}^{\text {tail }}$ & $A_{\mathrm{V}}^{90 a}$ & $\bar{A}_{\mathrm{V}}{ }^{b}$ & $M_{\text {tot }}\left[10^{4} M_{\odot}\right]^{b}$ & $\frac{M_{\text {tail }}}{M_{\text {tot }}}$ & $N_{\text {clumps }}$ \\
\hline \multicolumn{7}{|c|}{ Star-forming clouds, physical resolution $0.1 \mathrm{pc}$} \\
\hline Ophiuchus & 2.8 & 6.7 & 2.4 & 0.52 & 0.21 & 20 \\
\hline Taurus & 4 & 7.7 & 2.2 & 0.99 & 0.09 & 33 \\
\hline Serpens $^{c}$ & - & - & - & - & - & - \\
\hline Cha I & 2.1 & 3.2 & 1.7 & 0.27 & 0.16 & 11 \\
\hline Cha II & 2.1 & 3.2 & 1.8 & 0.11 & 0.17 & 7 \\
\hline Lupus III & 3 & 4.1 & 1.7 & 0.08 & 0.07 & 18 \\
\hline CrA cloud & 2.3 & 4.1 & 1.7 & 0.10 & 0.13 & 11 \\
\hline Lupus I & 2.0 & 3.3 & 1.6 & 0.13 & 0.12 & 16 \\
\hline LDN1228 ${ }^{d}$ & 2.1 & 3.1 & 1.5 & 0.15 & 0.09 & 4 \\
\hline Pipe $^{c}$ & - & - & - & - & - & - \\
\hline $\mathrm{LDN}_{13} 4^{d}$ & 2.2 & 3.7 & 1.5 & 0.09 & 0.07 & 3 \\
\hline $\mathrm{LDN} 204^{d}$ & 3.2 & 5.3 & 1.7 & 0.26 & 0.04 & 10 \\
\hline $\mathrm{LDN}_{1333^{d}}$ & 3.1 & 5.0 & 1.5 & 0.25 & 0.01 & 9 \\
\hline \multicolumn{7}{|c|}{ Non-star-forming clouds, physical resolution $0.1 \mathrm{pc}$} \\
\hline LDN1719 $9^{b}$ & - & - & 1.8 & 0.18 & - & - \\
\hline Musca & 2.0 & 3.2 & 1.7 & 0.04 & 0.16 & 3 \\
\hline Cha III & 2.1 & 5.5 & 1.7 & 0.12 & 0.11 & 26 \\
\hline Lupus V & - & - & 1.8 & 0.28 & - & - \\
\hline \multicolumn{7}{|c|}{ Star-forming clouds, physical resolution $0.6 \mathrm{pc}$} \\
\hline Ori A GMC & 3 & 5.6 & 2.2 & $9.2^{1}$ & 0.20 & 15 \\
\hline Per cloud & 3 & 4.9 & 2.0 & 1.4 & 0.12 & 10 \\
\hline Ori B GMC & 2.1 & 5.4 & 1.9 & 7.0 & 0.19 & 35 \\
\hline Cepheus A & 3.6 & 13 & 2.7 & 1.1 & 0.10 & 3 \\
\hline California & 4.2 & - & 1.7 & 10 & 0.01 & 14 \\
\hline
\end{tabular}

Notes. ${ }^{(a)}$ Extinction above which the contribution of the log-normal component to the PDF is less than $10 \%$. ${ }^{(b)}$ Calculated using the column density values above $A_{\mathrm{V}}>1$ mag. ${ }^{(c)} A_{\mathrm{V}}^{\text {tail }}$ could not be defined properly for the cloud. ${ }^{(d)}$ The most prominent Lynds Dark Nebula in the region.

the area, varies between $\sim 0.1-3$ pc. Figure 2 shows the size distribution of all clumps in all clouds, showing that smaller regions are always more numerous than larger ones down to the resolution limit of our data $(R \approx 0.1 \mathrm{pc})$. The size distribution has a power-law-like shape with the approximate slope of $-0.9 \pm 0.2$. Figure 2 also shows the mass distribution of the clumps, calculated by integrating Eq. (3) over the clump. The mass distribution covers roughly four orders of magnitudes between $10^{-1}-10^{3} M_{\odot}$, with a shape resembling a power-law distribution that has a slope of $-0.4 \pm 0.2$. This slope is flatter than typically observed for the mass distributions of cores in the clouds ( - 1.3, e.g. Motte et al. 1998; Alves et al. 2007; André et al. 2010), and is closer to the slopes derived for molecular clouds or $\mathrm{CO}$ clumps within individual clouds ( - 0.6, e.g. Williams et al. 1995; Kramer et al. 1998; Blitz et al. 2007). It is possible, however, that the derived slope is affected by the blending of clumps with each other. This blending can make the detection efficiency of clumps a function of the clump mass, and thereby affect the slope of the observed mass function (e.g., Kainulainen et al. 2009a; Pineda et al. 2009). The mass-to-size relation for the clumps, also shown in Fig. 2, roughly follows the relation $M \propto R^{2.7 \pm 0.2}$, which is again close to what has been derived for CO clumps within clouds (e.g., Carr 1987). The relation also agrees with what is expected for constant mean volume density spheres and it is steeper than predicted by Larson's relations $\left(M \propto R^{2}\right)$. But Larson's mean-density size relationship, $\rho \propto R^{-1}$ has been questioned by Ballesteros-Paredes \& Mac Low (2002), who suggested that it is an observational artifact owing to the limited dynamical range in column density. Our mass-radius relation, $M \propto R^{2.7 \pm 0.2}$, can also be seen as an indicator of the fractal dimension of the cloud, $D \approx 2.7 \pm 0.2$. This range is consistent with the highest values in the range, $D=2.3-2.7$, which was previously found by Elmegreen \& Falgarone (1996), indicating fairly space-filling column density structures (Sánchez et al. 2005; Federrath et al. 2009).

The mean volume densities in the clumps, defined as $\bar{n}=$ $M /\left(4 / 3 \pi R^{3}\right) / m_{\mathrm{H}}$, is on the order of $10^{3} \mathrm{~cm}^{-3}$. The mean volume densities of all clumps in all clouds have a distribution that peaks strongly at $\bar{n} \approx 0.8 \times 10^{3} \mathrm{~cm}^{-3}$ (shown in Fig. 2). The peak of the distribution is also relatively narrow, with the mean density being between $\bar{n}=0.4-2.1 \times 10^{3} \mathrm{~cm}^{-3}$ for $90 \%$ of the clumps. We also calculated the mean density of the diffuse component in the clouds. This is straightforwardly defined for each cloud without the clumpfinding process, because the diffuse component is always a uniform structure. Similarly with the clumps, the volumes of the clouds were calculated using the effective radii $R=\sqrt{A / \pi}$. The resulting mean densities, listed in Table 1 as well, are $\bar{n}_{\text {diff }}=1-2 \times 10^{2} \mathrm{~cm}^{-3}$, i.e. 5-10 times lower than the mean densities of the clumps identified from the dense component.

\subsubsection{Correlation with $\mathrm{CO}$ and linewidth}

In Fig. 3 we demonstrate how the spatial extent of the dense component compares to the common molecular line tracer observations. We use as an example the Ophiuchus and Perseus clouds for which large-scale ${ }^{12} \mathrm{CO}$ and ${ }^{13} \mathrm{CO}$ data are publicly available through the COMPLETE survey (Ridge et al. 2006a). Figure 3 shows the ${ }^{12} \mathrm{CO}$ total antenna temperature map of the Ophiuchus cloud with a contour of $A_{\mathrm{V}}^{\text {tail }}=2.8$ mag overplotted. In Ophiuchus, thresholding at $A_{\mathrm{V}}^{\text {tail }}$ separates two larger clumps: the main cluster region, and the streamers leading east from the cluster (there are additional clumps identified outside the coverage of the $\mathrm{CO}$ emission). In general, the $A_{\mathrm{V}}^{\text {tail }}$ contour coincides quite well with the extent of the ${ }^{12} \mathrm{CO}$ line emission data (1- $\sigma$ rms error of $\mathrm{CO}$ data is $0.98 \mathrm{~K}$ ), while ${ }^{13} \mathrm{CO}$ is spatially less extended. Figure 3 also shows a similar comparison on a smaller spatial scale for the B5 globule in the Perseus cloud.

We identified 10 clumps in the Ophiuchus and Perseus clouds that are fully within the region covered by the COMPLETE survey. We estimated the virial parameters of these clumps, defined as the ratio of kinetic-to-gravitational energies in the clump (Bertoldi \& McKee 1992):

$\alpha=\frac{5 \sigma^{2} R}{G M}$,

where $G=1 / 232 M_{\odot}^{-1} \mathrm{pc}\left(\mathrm{km} \mathrm{s}^{-1}\right)^{2}$ is the gravitational constant and $\sigma$ the velocity dispersion. The linewidths were estimated from both the ${ }^{12} \mathrm{CO}$ and ${ }^{13} \mathrm{CO}$ data by calculating the mean spectrum over the clump and fitting a simple Gaussian to its peak. The mass was calculated from the extinction data following Eq. (3). This calculation yielded virial parameters $\alpha=3-100$ for the clumps. The virial parameters correlate with the mass of the clumps approximately in a power-law fashion (Fig. 4). A simple linear least-squares fit to the data points yields the slopes of $-0.69 \pm 0.12$ and $-0.64 \pm 0.13$ for ${ }^{12} \mathrm{CO}$ and ${ }^{13} \mathrm{CO}$, respectively. This relation is consistent with the prediction for clumps confined by ambient pressure from their surrounding medium (Bertoldi \& McKee 1992), and this relation has been previously observed for clumps identified from CO emission data (e.g., Bertoldi \& McKee 1992; Williams et al. 1995; Lada et al. 2008). Clearly though, the determination of the virial parameters suffers from likely nongaussian errors, arising most pressingly from the uncertainty in determining the linewidth that would well trace 

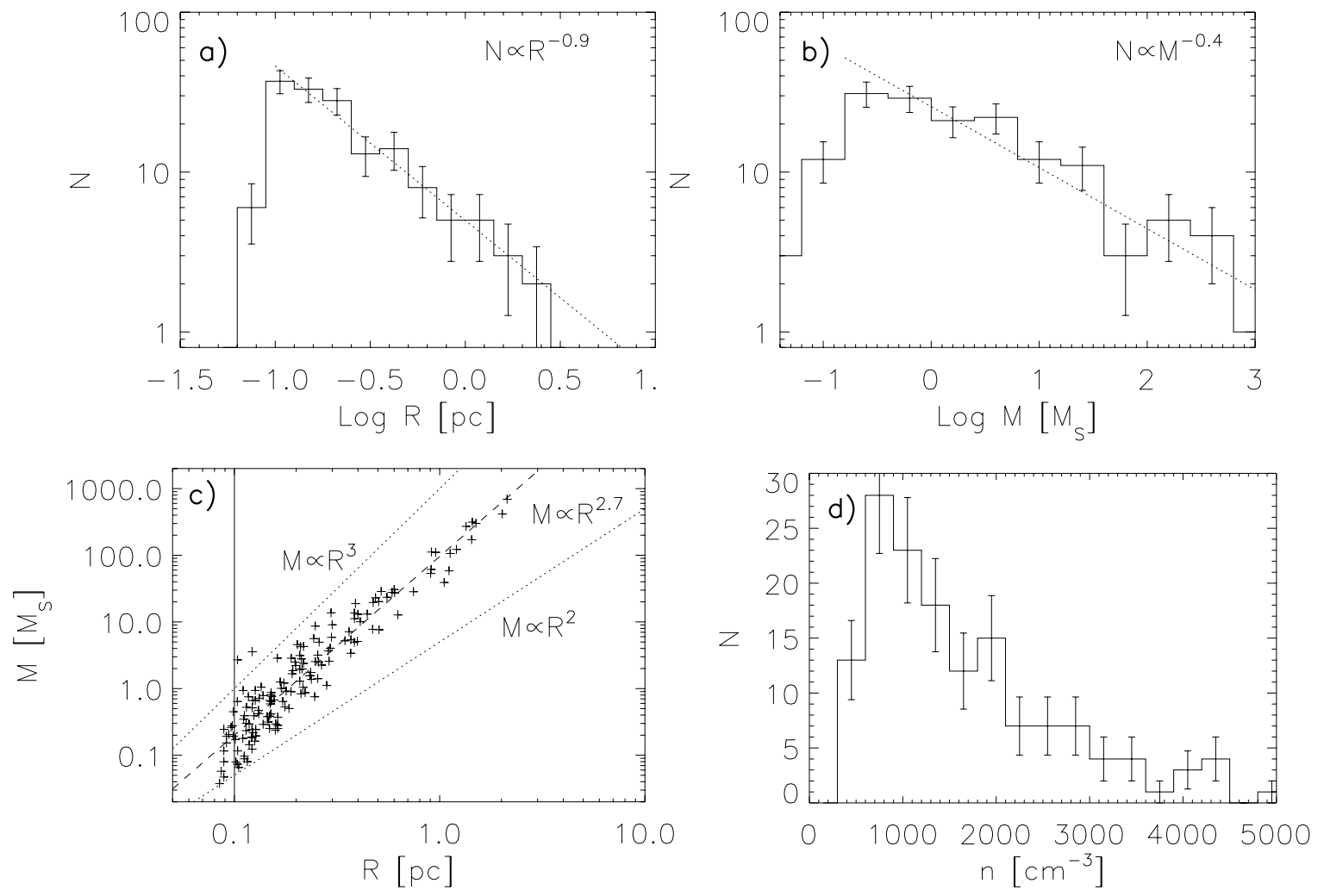

Fig. 2. Characteristics of the structures (clumps) defined by a thresholding at $A_{\mathrm{V}}^{\text {tail }}$ (see Sect. 3.1.1 in text). a) The size distribution. Error bars show the $\sqrt{n}$ uncertainty. The dotted line shows the least-squares fit to the distribution, with the slope $-0.9 \pm 0.2$. b) The same for the mass distribution. The linear fit results in the slope $-0.4 \pm 0.2$. c) The mass-radius relation. Overplotted are slopes indicating $M \propto R^{3}$ and $M \propto R^{2}$ (dotted lines), the linear fit to the data points which has a slope $2.7 \pm 0.2$ (dashed line), and the resolution limit $R=0.1 \mathrm{pc}$ of the data (solid line). d) The distribution of mean volume densities.

most of the gaseous material in the cloud. Therefore, we consider this observed correlation indicative, although clearly not well constrained. It is, however, evident that having $\alpha \gg 1$, these clumps are not gravitationally bound entities (although they can be significantly supported by other forces, as will be discussed later). This is unsurprising, because $\mathrm{CO}$ clumps in molecular clouds are generally observed to have high virial parameters (e.g., Carr 1987; Bertoldi \& McKee 1992; Falgarone et al. 1992).

Figure 4 also shows the size-linewidth relation for the clumps in Ophiuchus and Perseus. The data are scattered with no clear correlation, although similarly with the virial parametermass relation determining the correlation is hampered by the small number of clumps. We note that if the size and linewidth are uncorrelated, the relation between the virial parameter and mass established above $\left(\alpha \propto M^{-2 / 3}\right)$ implies that $M \propto R^{3}$, i.e. the mean volume densities of the clumps are constant (see Eq. (4)). Indeed, this agrees with the characteristics of the clumps derived in Sect. 3.1.2, i.e., that the mass-radius relation approximately follows $M \propto R^{2.7 \pm 0.1}$, and that the mean volume densities of the clumps strongly peak around a characteristic value of $\sim 10^{3} \mathrm{~cm}^{-3}$.

\subsection{Pressure confinement of molecular clumps}

As demonstrated in Sect. 3.1.1, the mass in the clumps above $A_{\mathrm{V}}^{\text {tail }}$, i.e., in the dense component, accounts only for the minor fraction of the total gaseous mass of a cloud. In other words, the clumps are surrounded by a medium whose total mass (and spatial extent) greatly exceeds that of their own. As an example, the most massive clump in our cloud sample has about $10 \%$ of the mass of the whole cloud (for other clumps, the fraction is much smaller). Likewise, as shown in Sect. 3.1.2, the mean density of the clumps is almost an order of magnitude higher than the mean density of their surrounding medium. Thus, it seems reasonable to consider the external pressure from the surrounding medium as a force that supports the clumps against dispersal. Below, we follow the formulation of Bertoldi \& McKee (1992) and examine the scale of external support provided to the clumps by the diffuse surrounding medium.

The basic condition for the virial balance of a clump is

$0=W+2\left(T-T_{\text {ext }}\right)+\mathcal{M}$.

In this equation, $W$ is the potential energy

$W=-\frac{3}{5} \frac{G M^{2}}{R}$

and $T$ and $T_{\text {ext }}$ are the kinetic energy of the clump and its surface term

$T=3 / 2 \times P_{\text {kin }} V$,

$T_{\text {ext }}=3 / 2 \times P_{\text {ext }} V$.

$\mathcal{M}$ is the magnetic energy which we neglect for simplicity. The virial balance equation expressed in terms of pressure is then

$P_{\text {kin }}=P_{\text {gr }}+P_{\text {ext }}$.

In this, the total kinetic pressure of the clump, $P_{\text {kin }}$, is the sum of both thermal and nonthermal components:

$P_{\text {kin }}=\rho\left(\sigma_{\mathrm{T}}^{2}+\sigma_{\mathrm{NT}}^{2}\right)$, 

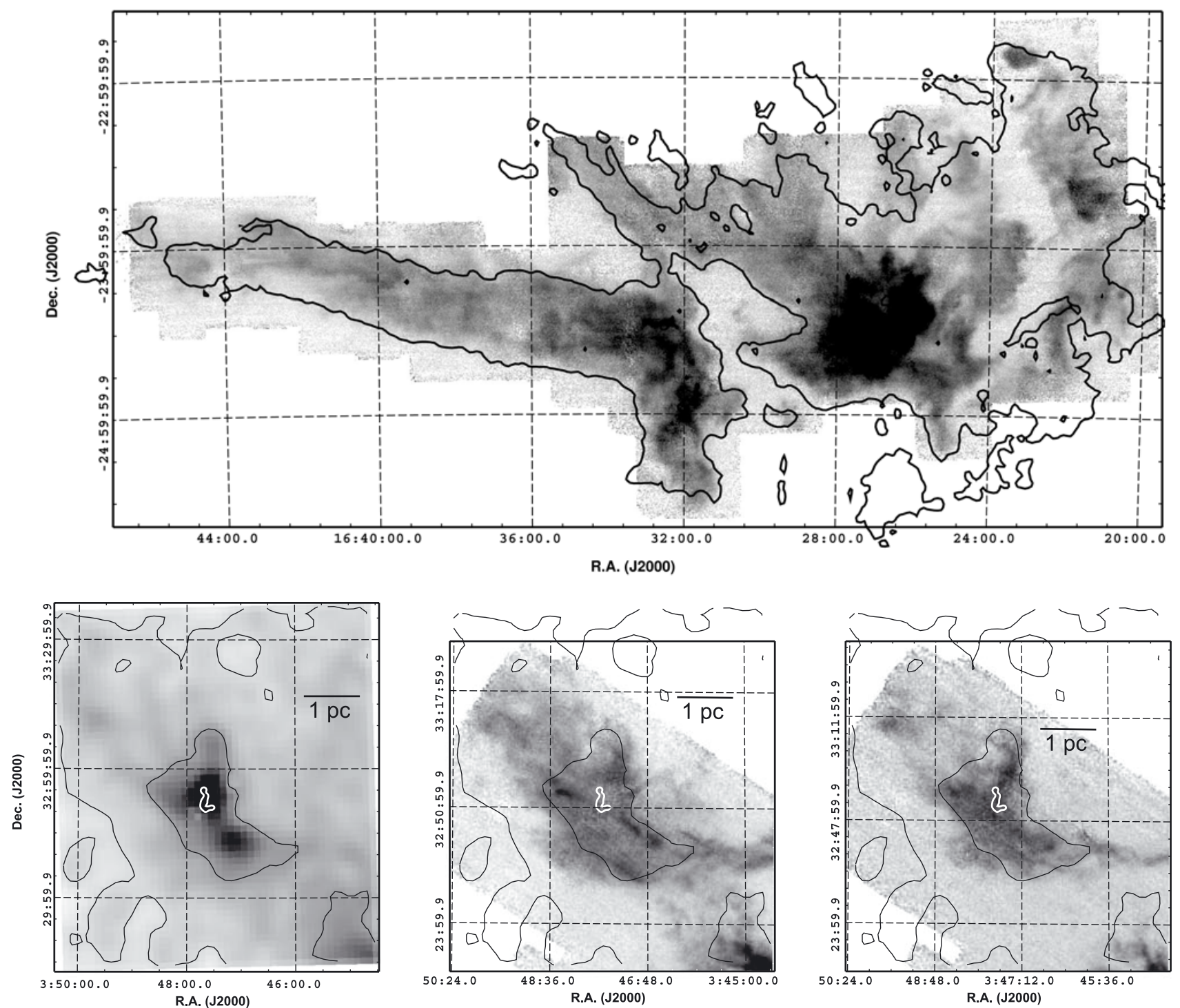

Fig. 3. Comparison of the dense component, i.e., structures above $A_{\mathrm{V}}>A_{\mathrm{V}}^{\text {tail }}$, with the CO molecular line emission. Top: ${ }^{12} \mathrm{CO}$ line emission from the COMPLETE survey for Ophiuchus, with a contour of $A_{\mathrm{V}}^{\text {tail }}=2.8$ mag overplotted. Bottom row: similar comparison for the B5 globule in Perseus. The left panel shows the extinction map, with black contours at $A_{\mathrm{V}}=[1,3]$ mag. The white contour shows the extent of the coherent core in which the linewidth of the $\mathrm{NH}_{3}$ molecule emission drops abruptly, identified by Pineda et al. (2010a). The center and right panels show the same for the ${ }^{12} \mathrm{CO}$ and ${ }^{13} \mathrm{CO}$ line emission, respectively.

and $P_{\mathrm{gr}}$ is the gravitational pressure of the clump supporting it against expansion:

$P_{\mathrm{gr}}=-1 / 3 \times W / V=\left(\frac{4 \pi}{15}\right) G(\bar{\rho} R)^{2}$

$P_{\text {ext }}$ is the external pressure on the clump. Under the assumption that molecular cloud complexes are close to gravitational virial equipartition (e.g., Larson 1981; Heyer et al. 2001), a supporting external pressure is directed at a clump. This pressure arises from the turbulent pressure that balances the cloud against its own gravity. Because the cloud, as a whole, is close to virial equipartition, the turbulent pressure amounts to the gravitational pressure of the cloud (analogously to Eq. (11)), but we adopt a slightly modified expression that takes into account that the cloud is not spherical (Bertoldi \& McKee 1992). With the definition of the mean mass surface density, $\bar{\Sigma}=M /\left(\pi R^{2}\right)$, the external pressure supporting clumps against dispersal is

$P_{\mathrm{ext}}=P_{\mathrm{gr}}^{\text {cloud }}=\left(\frac{3 \pi a_{1}}{20}\right) G \bar{\Sigma}^{2} \phi_{\mathrm{G}}$,

where $a_{1}$ and $\phi_{\mathrm{G}}$ are numerical constants related to the cloud morphology whose value can be evaluated as prescribed in Bertoldi \& McKee (1992). As an example of the order-ofmagnitude of these pressures, using the typical ${ }^{13} \mathrm{CO}$ linewidth of $\sigma=0.75 \mathrm{~km} \mathrm{~s}^{-1}$ for a $R=1 \mathrm{pc}$ sized clump, $\bar{n}_{\text {diff }}=150 \mathrm{~cm}^{-3}$, $\bar{n}_{\text {clump }}=800 \mathrm{~cm}^{-3}$, and the mean mass surface density $\bar{A}_{\mathrm{V}}=$ 2 mag yields the pressure ratios $P_{\text {kin }} \approx 10 \times P_{\mathrm{gr}} \approx 4 \times P_{\text {ext }}$. In other words, the pressures supporting the clumps against dispersal amount in total to about one third of the pressure driving their dispersal.

Below, we examine these pressures for a population of clumps whose properties equal those derived for the clumps identified in this paper. In Sect. 3.1.3 we showed that the observed virial parameters of the clumps scale with their masses 

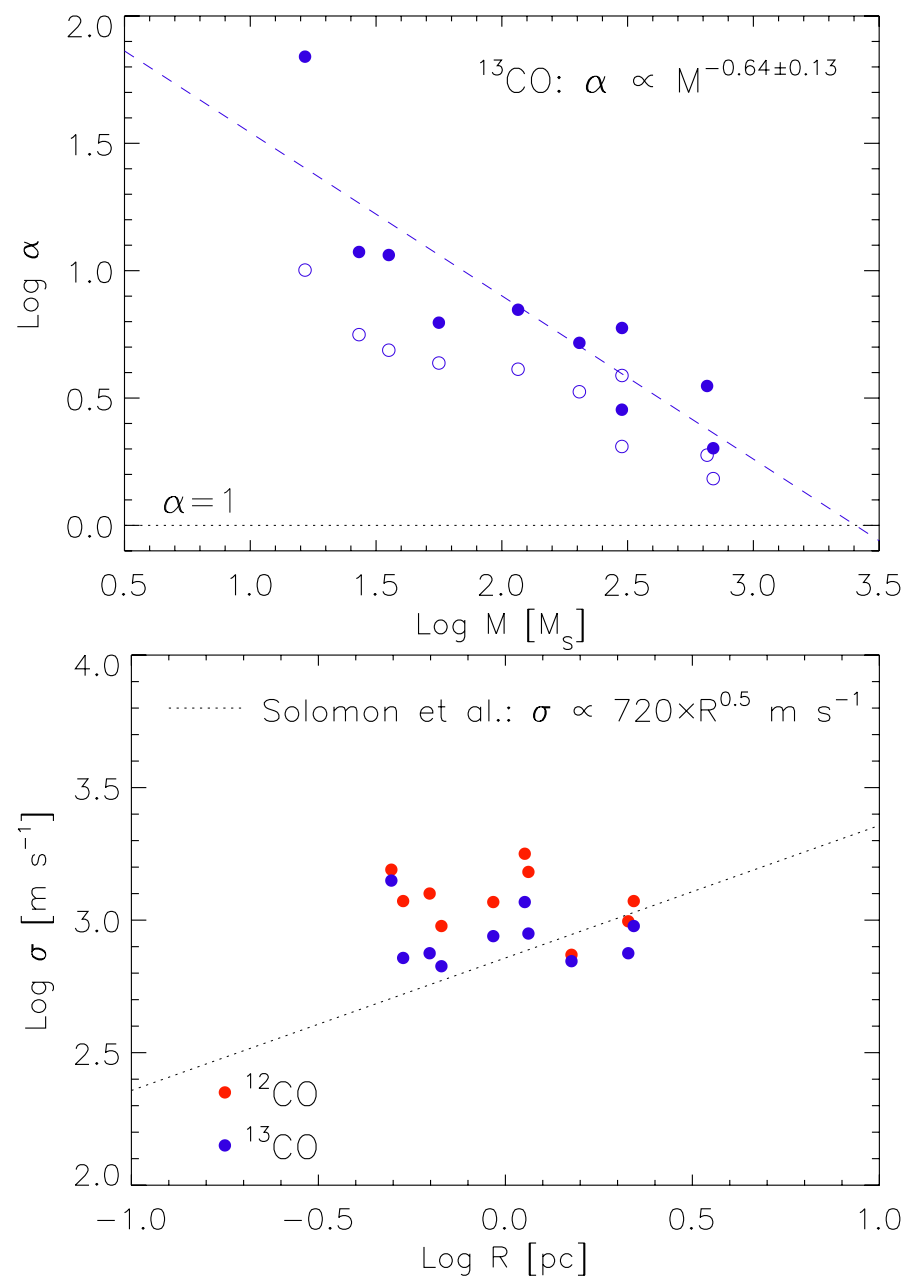

Fig. 4. Top: virial parameters derived for clumps identified in the Ophiuchus and Perseus clouds using thresholding at $A_{\mathrm{V}}=A_{\mathrm{V}}^{\text {tail }}$. In calculating the virial parameter, linewidths derived from ${ }^{13} \mathrm{CO}$ data from the COMPLETE survey (Ridge et al. 2006a) were used. The dashed line shows the slope corresponding to the relation $\alpha \propto M^{-2 / 3}$, predicted for clumps confined by external pressure (Bertoldi \& McKee 1992). A linear fit to the data points yields the slope $-0.64 \pm 0.13$. The open circles show the modified virial parameters of the same clumps, $\widetilde{\alpha}$, that take into account the external confining pressure (see Sect. 3.2 in text). The dotted line indicates the $\alpha=1$ (and $\widetilde{\alpha}=1$ ) level. Bottom: sizelinewidth relation for the same clumps, shown for both ${ }^{12} \mathrm{CO}$ (red) and ${ }^{13} \mathrm{CO}$ (blue). The dotted line shows the $\sigma \propto R^{0.5}$ relation (Solomon et al. 1987), not a fit to the data points.

(which is predicted for clumps confined by external pressure, Bertoldi \& McKee 1992):

$\alpha=\frac{2 T}{|W|}=\frac{P_{\mathrm{kin}}^{\text {clump }}}{P_{\mathrm{gr}}^{\text {clump }}}=c_{1} \times M^{-2 / 3}$,

where $c_{1}$ is a proportionality constant. It directly follows from this dependence that the internal kinetic pressure of the clumps is only a function of their density (Eqs. (13) and (11)), and thereby the kinetic pressure is constant for a population of constant density clumps. The ratio of outward to inward pressures for a clump is then

$$
\frac{P_{\text {out }}^{\text {clump }}}{P_{\text {in }}^{\text {clump }}}=\frac{P_{\text {kin }}}{P_{\text {ext }}+P_{\mathrm{gr}}}=\frac{c_{1}\left(\frac{4 \pi}{3}\right)^{-2 / 3}}{(\bar{\rho})^{2 / 3} R^{2}+\frac{9 a_{1} \phi_{\mathrm{G}}}{16} \frac{(\bar{\Sigma})^{2}}{(\bar{\rho})^{4 / 3}}} .
$$

Figure 5a illustrates this ratio (Eq. (14)) as a function of the mean mass surface density $\bar{\Sigma}$ (in units of $A_{\mathrm{V}}$ ) for clumps of different sizes $(R=0.1-2.1 \mathrm{pc})$. As the mean density we used the value $\bar{n}=800 \mathrm{~cm}^{-3}$ shown above to be the peak of the mean densities in the clumps (Sect. 3.1.2). Figure 5 shows that the transition from a regime where structures are unbound to a regime where they are bound occurs around $\bar{A}_{\mathrm{V}} \approx 4 \operatorname{mag}\left(\bar{\Sigma} \approx 80 \mathrm{M}_{\odot} \mathrm{pc}^{-2}\right)$ for clumps over a wide range of sizes $(R=0.1-2.1 \mathrm{pc})$. This value is higher than the observed mean mass surface densities by a factor of about $\sim 2$ (see Table 1), and therefore, the external pressure is lower than the internal kinetic energy of the clump by a factor of $\sim 4$.

We so far assumed that the kinetic energies of the clumps scale with mass as shown by Eq. (13). In principle, this scaling is supported by the linewidth data of clumps, which show fairly constant linewidths (Fig. 4). This observation is, however, hampered by the poor statistics we could achieve with the available data. Therefore, we also consider a case where the kinetic energies of the clumps scale according to a Larson-like sizelinewidth relation (Solomon et al. 1987):

$\alpha^{\text {clump }}=\frac{2 T}{|W|}=\frac{P_{\mathrm{kin}}^{\text {clump }}}{P_{\mathrm{gr}}^{\text {clump }}}=\frac{5 \times 0.72\left(\frac{R}{1 \mathrm{pc}}\right)^{0.5} R}{G M}$.

Again, Fig. 5b shows the ratio of outward to inward pressures for clumps (Eq. (14)) as a function of mean mass surface density of the cloud. Using this scaling of kinetic energies, the balance occurs approximately at the level of the observed mean mass surface density $\left(\bar{A}_{\mathrm{V}} \approx 2 \mathrm{mag}\right)$. While our observations seem to favor kinetic energy scaling with mass (see also discussion in Sect. 4), this example illustrates the behavior of the pressure balance in another plausible scaling scheme, suggesting that the external pressure can indeed be close to the internal kinetic energy of the clumps.

Finally, we illustrate the net effect of the pressures on the individual observed clumps by calculating modified virial parameters for the clumps for which we have CO data. In this modified virial parameter, we take into account the external pressure on the clumps:

$$
\widetilde{\alpha}^{\text {clump }}=\frac{P_{\text {kin }}^{\text {clump }}}{P_{\mathrm{gr}}^{\text {clump }}+P_{\text {ext }}} .
$$

The modified virial parameters are shown in Fig. 4 together with the traditional virial parameters that include only the gravitational and kinetic energies of the clumps. In agreement with the earlier results, the modified virial parameters are clearly smaller compared those resulting from Eq. (4), but they are still somewhat larger than unity.

\section{Discussion}

\subsection{Pressure confinement of the clumps}

We described in the previous section a new approach for characterizing structures observed in molecular clouds. In particular, we used the observed gas column density PDFs of molecular clouds to define a population of clumpy structures (dense component) embedded in the extended, interclump medium (diffuse component). The transition between these components occurs at the extinction threshold $A_{\mathrm{V}}^{\text {tail }}=2-4 \mathrm{mag}$, or equivalently, at $\Sigma^{\text {tail }}=40-80 M_{\odot} \mathrm{pc}^{-2}=0.008-0.017 \mathrm{~g} \mathrm{~cm}^{-2}$. This level is relatively constant and is in the quoted range in every cloud 

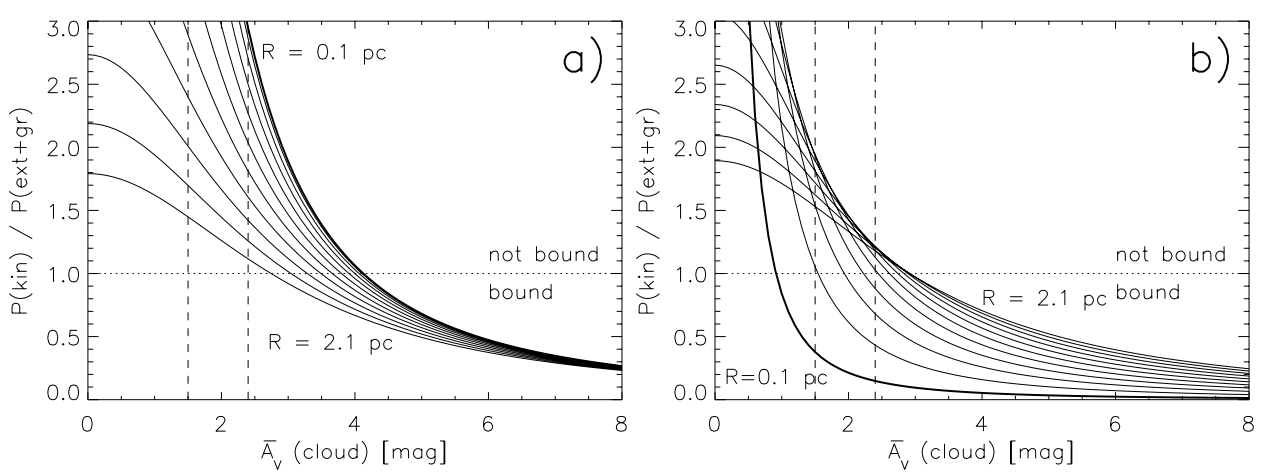

Fig. 5. a) Ratio of the pressures supporting a clump against a collapse (total kinetic pressure) to the pressures promoting it (external and gravitational pressure) in clumps with $\bar{n}=800 \mathrm{~cm}^{-3}$. The different curves are for clumps with radii $R=0.1-2.1 \mathrm{pc}$, in steps of $0.2 \mathrm{pc}$. The dashed vertical lines indicate the interval of the observed mean extinctions (see Table 1). b) The same, but using Larson's size-linewidth relation to calculate the kinetic energies of the clumps (i.e., Eq. (15) instead of Eq. (13)).

except one (Serpens, cf. Table 1), for which it could be reliably determined. The dense component becomes dominant at $A_{\mathrm{V}}=3-8 \mathrm{mag}$. The mass of the dense component is between $1-20 \%$ of the total mass of the cloud, which we defined as the total mass above $A_{\mathrm{V}}>1$ mag. The clumps of the dense component show roughly power-law-like distributions of sizes and masses, covering wide dynamical ranges (see Fig. 2). However, the components are characterized by remarkably constant mean volume densities of $\bar{n} \approx 10^{3} \mathrm{~cm}^{-3}$ and $\bar{n} \approx 1.5 \times 10^{2} \mathrm{~cm}^{-3}$ for the dense and diffuse components, respectively.

The clumps identified using the column density PDFs of the clouds in this study are very similar to the gravitationally unbound ${ }^{13} \mathrm{CO}$ clumps identified in several studies in the past (e.g. Carr 1987; Bertoldi \& McKee 1992; Williams et al. 1995; Lada et al. 2008). In particular, the mean densities and mass-radius and virial parameter-mass relations derived in Sects. 3.1.2, 3.1.3 agree to what has been derived for such CO clumps. Similarly, those studies have concluded that the external pressure can be a significant confining source for these clumps. A simple qualitative comparison between the clumps defined with the $A_{\mathrm{V}}^{\text {tail }}$ threshold and ${ }^{13} \mathrm{CO}$ clumps indeed suggests that these approaches may trace quite similar components in the clouds (see Fig. 3).

It has been suggested previously (Goodman et al. 1998; Caselli et al. 2002) and was more recently directly observed (Pineda et al. 2010a) that there appears to be a sharp transition to dynamically coherent objects, or cores, at the scale where nonthermal motions cascade from the supersonic to the subsonic regime (the sonic scale, e.g. Vázquez-Semadeni et al. 2003; Federrath et al. 2010). In particular, Pineda et al. (2010a) detected such a transition in the B5 globule in the Perseus cloud, at the length scale on the order of $R \approx 0.1 \mathrm{pc}$, in agreement with the sonic scale (and Larson's size-linewidth relation). The spatial extent of this coherent core is illustrated in Fig. 3 together with the extent of the clump defined by $A_{\mathrm{V}}^{\text {tail }}$. The transition to coherence clearly occurs in a different column density regime than the break in the column density PDF defined by $A_{\mathrm{V}}^{\text {tail }}$. Importantly, while the structural transition from supersonic- to subsonic velocities in cloud structure seems to be linked to a particular sizescale, the structural transition described by the $A_{\mathrm{V}}^{\text {tail }}$ threshold is not size-dependent. As shown in Fig. 2, structures above $A_{\mathrm{V}}^{\text {tail }}$ cover a large size- and mass range, with their number decaying in a roughly power-law-like fashion. Thus, the $A_{\mathrm{V}}^{\text {tail }}$ threshold appears to be unrelated to the transition to coherent cores in the velocity structure. Unfortunately, the column density maps used in our work do not provide sufficiently high spatial resolution to properly sample the PDF at the length scale of the transition to coherence. Therefore, we were unable to directly look for possible features that the transition would induce to the PDFs.

We showed in Sect. 3.2 that a significant external pressure from the surrounding cloud is imposed on the clumps we identified using the $A_{\mathrm{V}}^{\text {tail }}$ threshold. This is especially the case if, instead of a constant kinetic pressure, clumps follow a Larson-like size-linewidth scaling relation. This indicates that the clumps may even be close to a pressure balance with their surroundings. The $\mathrm{CO}$ linewidths and virial parameters we derived for a small sample of clumps partially support this picture: virial parameters (as defined by Eq. (4)) correlate with clump masses as predicted for pressure-confined clumps, and the linewidths show no clear correlation with clump sizes (we observe a nearly constant linewidth for the clumps). On the other hand, the modified virial parameters that take the external pressure into account (Fig. 4 and Eq. (16)) were somewhat in excess to unity for those clumps, implying that they may be over-pressurized and thus either an additional pressure component may be significantly affecting them, or they may be expanding. This result is, again, similar to what has been derived for ${ }^{13} \mathrm{CO}$ clumps (e.g., Carr 1987).

The role of the internal gravitational pressure of the clumps is further illustrated in Fig. 6, which shows the mean density for all identified clumps as a function of the clump size (plus signs in the figure). A constant ratio of gravitational-to-external pressures defines a linear relationship in this plot with a slope of -1 (cf. Eqs. (11) and (12)). The relation corresponding to $P_{\mathrm{gr}}=$ $P_{\text {ext }}$ is overplotted in Fig. 6. All clumps identified from the dense component have densities lower than this relation, implying that the gravitational energy is indeed low compared to the external pressure. The typical mean density $n=150 \mathrm{~cm}^{-3}$ of the diffuse component is also shown, which obviously is clearly below the mean densities of the clumps.

Despite the limits imposed by the spatial resolution and dynamical range of the extinction maps, we can still examine the role of gravity in the structures nested inside the clumps (i.e., in smaller-scale structures inside of what we defined as a clump). We show in Fig. 6 with red diamonds a population of structures identified by an experiment in which we defined clumps using a threshold level $A_{\mathrm{V}}=3 \times A_{\mathrm{V}}^{\text {tail }}$. While this threshold is typically $A_{\mathrm{V}}=6-12 \mathrm{mag}$, this selection likely represents a population connected to star-forming regions, or at least, pre-stellar objects. The structures identified with this experiment are above the 
$P_{\mathrm{gr}}=P_{\text {ext }}$ line $^{1}$. This demonstrates how gravitation becomes an increasingly important confining force for density enhancements nested inside the clumps. To illustrate one case where gravitation is known to eventually become the dominant force, we marked in the diagram the star-forming clump B5 in Perseus (see Fig. 3). The clump defined by thresholding at $A_{\mathrm{V}}^{\text {tail }}$ is marked with a black filled circle, and the structure identified inside it with the threshold at $3 \times A_{\mathrm{V}}^{\text {tail }}$ is marked with a red filled circle.

Given these results, we suggest that the observed organization of structures, identified with our new approach in using the column density PDF, can be understood as a population of clumps that are significantly supported by the external pressure. This external pressure originates from the turbulent pressure outside the clumps, and in this framework, it is a consequence from the assumption that clouds as a whole are close to virial equipartition. Then, the break observed in the column density PDFs at $A_{\mathrm{V}}^{\text {tail }}$ represents a transition from a diffuse inter-clump medium to clumps significantly supported by external pressure. This interpretation has some profound implications. Most pressingly, it implies that the external pressure from the large-scale cloud has an important role in the formation of molecular cloud structures over wide size and mass scales. This result is analogous to the recent work of Lada et al. (2008), who found the external pressure a significant force in confining small-scale cores, or globules, in the Pipe Nebula. We note that we speculated in Paper I that the transition in the PDFs could be related to a transition from a regime where gravitation is negligible to a regime where it is dominant. The results of this paper, however, revise this picture and more quantitatively connect the break to the pressure conditions of density enhancements with their surroundings.

We emphasize three main assumptions of this framework. First, we assume that molecular clouds are in virial equilibrium as a whole, in order to relate their gravitational pressure to the turbulent pressure confining the clumps. Although close to being in virial equilibrium, molecular clouds are also embedded in an external medium and may have been formed in large-scale converging flows, which are highly dynamical objects, where surface terms play an important role (see e.g., Ballesteros-Paredes 2006; Banerjee et al. 2009; Schneider et al. 2010). Second, as can be seen from Fig. 5, the external pressure due to the turbulent pressure has a significant role in providing support for the clumps. The confining force exerted to a clump by it, however, depends on the isotropy of the turbulent flow surrounding the clump. Supersonic turbulence is highly anisotropic locally and will lead to transient formation and destruction of filaments and clumps. While estimating the net effect of the turbulent pressure in a more consistent way for each clump would require more detailed velocity information (and modeling of the cloud structure) than are available for this work, we estimated the average level the pressure may have on clumps implicitly in Eq. (12). Third, we neglected the magnetic field stress in our approach. The exact role of the magnetic fields in shaping cloud morphology is still under debate, but generally, it can provide confining pressure perpendicular to the field lines which supports clumps against both collapse and expansion. The magnitude of this support can be estimated roughly with $B^{2} /(8 \pi)$, which generally leads to pressures similar to turbulent ram pressures for the field strengths of $B \approx 15 \mu \mathrm{G}$ (Crutcher 1999).

\footnotetext{
1 This relation does not depend on the pressure balance or virial status of the clumps (see Eqs. (11) and (12)). To estimate the kinetic pressure of the clumps identified in this experiment, a tracer probing the densities of these objects would have to be used instead of ${ }^{12} \mathrm{CO}$ or ${ }^{13} \mathrm{CO}$.
}

Interestingly, Crutcher et al. (2010) recently found that at densities lower than $n \lesssim 300 \mathrm{~cm}^{-3}$ the magnetic field strength do not scale with density, implying that below that density the cloud material is likely channelled along the field lines. Above this threshold density, the field strength approximately scales with density as $B \propto n^{0.65}$. In the context of our work, the threshold density of $n \approx 300 \mathrm{~cm}^{-3}$ is in the regime between the diffuse component $\left(\bar{n} \approx 150 \mathrm{~cm}^{-3}\right)$ and the clumps $\left(\bar{n} \approx 800 \mathrm{~cm}^{-3}\right)$. This raises the interesting possibility that the break in the column density PDFs at $A_{\mathrm{V}}^{\text {tai }}$ would be related to the change in the role of the magnetic field from dominant at low column densities to less significant at higher column densities. While Crutcher et al. (2010) suggests that this could be the regime where cloud structures become self-gravitating, our work rather suggests that this change in the $B-n$ relation could be caused by a transition to structures that are not self-gravitating but confined because of their approximate pressure balance with their surroundings.

\subsection{Pressure confinement and star formation}

When coupled with the main result of Paper I, i.e., that non-starforming clouds do not exhibit similarly strong tails (if any) in their PDFs as all star-forming clouds do, the interpretation discussed in this paper leads to a picture in which the formation of pressure-confined clumps occurs in clouds prior to (or at a clearly higher rate than) the formation of gravitationally dominated cores. Indeed, in our sample of molecular clouds, pressure confined clumps are observed in some clouds that do not show active star formation (Musca, Cha III), or even high column density cores (Musca et al., in prep.). This picture is further supported by the recent analysis of the stability of dense cores in the nearby, mostly quiescent Pipe Nebula (Lada et al. 2008). In this cloud, Lada et al. examined a sample of $\sim 150$ cores of masses between $0.2-20 M_{\odot}(R \approx 0.04-0.2 \mathrm{pc})$. They concluded the core population in the Pipe to be pressure-confined, gravitationally unbound entities. The cores in the Lada et al. study were defined to be single-peaked (or at most a-few-peaked) entities, and thereby they may well represent the smallest scale of the hierarchy whose largest scale is represented by the structures identified in our study. A similar result highlighting the role of external pressure in a star-forming cloud was recently published by Maruta et al. (2010). They investigated the stability of dense cores in the Ophiuchus cluster, in the regime of $R \lesssim 0.1$, and concluded that the external pressure has a significant role in the dynamics of the cores. These results clearly indicate that the hierarchy of structures nested inside clumps is affected by the external pressure all the way to the regime of dense star-forming cores.

In the interpretation discussed above, the formation of pressure bound clumps can be seen as a prerequisite for the formation of gravitationally bound cores. Given this, there evidently should be a relation between the occurrence of these clumps and star formation, even beyond the general observation that not all quiescent clouds show such clumps, while all star-forming clouds do. Therefore, it is interesting to consider the observed star-forming efficiencies and -rates in the clouds of our sample.

Recently, Heiderman et al. (2010) studied the star-forming activities of nearby molecular clouds as a function of the gas surface density (i.e., the Kennicutt-Schmidt law). In their work, Heiderman et al. used near-infrared extinction maps similar to those employed in this paper to derive gas surface densities. They examined the number of young stellar objects (YSOs) in the clouds identified using the Spitzer satellite data in different column density intervals and constructed the Kennicutt-Schmidt 


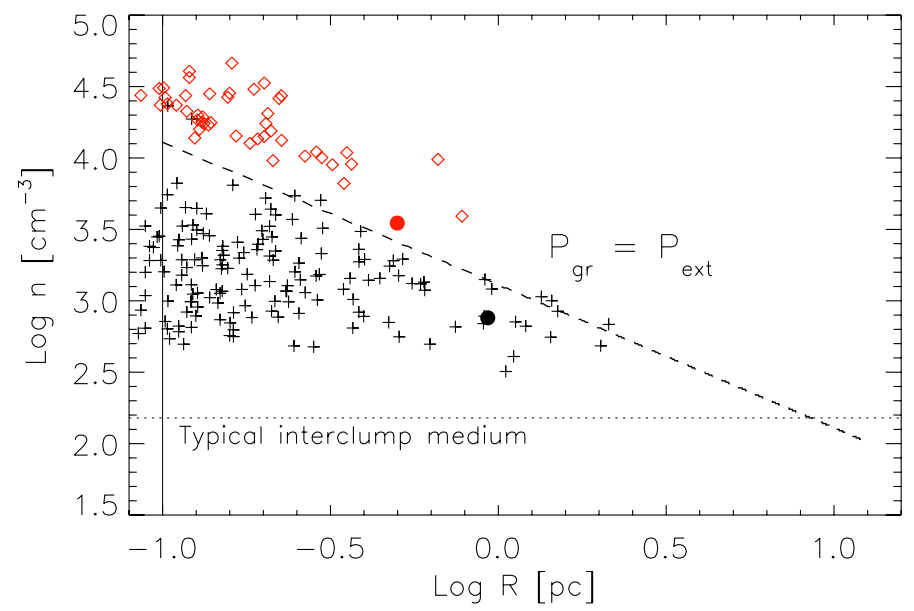

Fig. 6. Relation between the mean density and size of the structures (clumps) defined by thresholding at $A_{\mathrm{V}}^{\text {tail }}$. The dotted line shows the typical density of the interclump medium (i.e. the density of the diffuse component). The dashed line shows the density above which the gravitational energy of the clump becomes higher than the external surface pressure $\left(P_{\mathrm{gr}}=P_{\mathrm{ext}}\right)$. The red diamonds show the structures identified from the column density maps by thresholding at $A_{\mathrm{V}}=3 \times A_{\mathrm{V}}^{\text {tail }}$. The resolution limit of the column density data is at $\log R=-1.0$. The black solid circle marks the clump B5 in Perseus, and similarly, the red solid circle marks the same clump when using the threshold of $A_{\mathrm{V}}=3 \times A_{\mathrm{V}}^{\text {tail }}$.

law for their cloud sample. In particular, they observed an abrupt drop in the star-formation rate at $\Sigma \approx 50-100 M_{\odot} \mathrm{pc}^{-2}$ (see Figs. 3 and 8 in Heiderman et al. 2010), which lead them to suggest a threshold for star formation at $\Sigma_{\text {th }}=129 \pm 14 M_{\odot} \mathrm{pc}^{-2}$ $\left(A_{\mathrm{V}}=8.6 \mathrm{mag}\right)$. A very similar result was recently reached by Lada et al. (2010), who examined the relation between the number of YSOs in nearby clouds and the amount of high column density material in them. They showed that the correlation between the mass of the gas and the number of YSOs identified in the clouds is strongest (i.e., the dispersion in the relation is lowest) at $A_{\mathrm{K}} \approx 0.8 \mathrm{mag}\left(A_{\mathrm{V}} \gtrsim 7.3 \mathrm{mag}\right.$, or $\left.\Sigma \approx 116 M_{\odot} \mathrm{pc}^{-1}\right)$. We note that the dispersion of the SFR-surface density relation derived by Lada et al. (2010) starts to decrease already at surface densities lower than $\Sigma \approx 116 M_{\odot} \mathrm{pc}^{-1}$, reaching its minimum at that point.

The star-formation thresholds derived in the studies above are slightly higher than the typical $A_{\mathrm{V}}^{\text {tail }}$ values. However, we defined the $A_{\mathrm{V}}^{\text {tail }}$ value as the point where the dense component, on average, becomes a significant excess over the diffuse component. Obviously, at this surface density the largest contribution to the PDF still comes from the underlying diffuse component, not from the excessive dense component. Typical surface density values at which the contribution of the tail to the PDF becomes dominant $(>90 \%)$ are around $A_{\mathrm{V}}^{\text {tail }}(90 \%) \approx 3-8$ mag (listed in Table 1). These values would very well agree with the threshold values derived by Lada et al. (2010) and Heiderman et al. (2010), although very different approaches were used in these papers. Therefore, it seems plausible to interpret the increase in star-forming activity to be related to the regime where the column density PDF is becoming completely dominated by the dense component.

In the context of clumps bound by external pressure, a natural threshold for star formation is introduced by the surface density at which pressure-bound clumps form, which is around $A_{\mathrm{V}}^{\text {tail }}$. Furthermore, as discussed above, the mass above this threshold is in a direct connection to the SFR of the cloud, with the SFR increasing in a power-law manner with increasing gas surface density. This interpretation provides a physically motivated explanation for the star-formation threshold occurring at relatively low surface densities and links it to an observed structural feature in the clouds. Thus, we suggest a picture in which the formation of pressure-bound clumps, and thereby the structural transition at $A_{\mathrm{V}}^{\text {tail }}$, introduces a prerequisite for star formation, with the amount of mass in clouds above that limit directly proportional to the capability of the cloud to form stars.

\section{Conclusions}

We presented an analysis of the large-scale, clumpy structures in nearby molecular clouds and of their stability. In particular, we described a new way of identifying the structure in clouds using the observed column density PDFs. With this approach, we identified two distinctive components in them, referred to as the dense and diffuse components, and described their basic physical characteristics. We then examined the stability of the clumps in the dense component, especially by considering the scale of external pressure imposed on them by the surrounding medium. The main conclusions of our work are:

1. The transition between the diffuse and dense components occurs at a narrow range of column densities, $A_{\mathrm{V}}^{\text {tail }}=2-4 \mathrm{mag}$, or $\Sigma^{\text {tail }}=40-80 M_{\odot} \mathrm{pc}^{-2}$. The dense component dominates the observed column density PDFs above $A_{\mathrm{V}}>3-8 \mathrm{mag}$. The total mass of the dense component is $1-20 \%$ of the total mass of the cloud, and thus always clearly smaller than the mass of the diffuse component. Clumps identified in the dense component show wide dynamical ranges of sizes $(0.1-3 \mathrm{pc})$ and masses $\left(10^{-1}-10^{3} M_{\odot}\right)$. However, the mean volume density of the clumps is remarkably constant, $\bar{n} \approx 10^{3} \mathrm{~cm}^{-3}$. This is $\sim 5-10$ times higher than the mean volume density of the diffuse component, $\bar{n} \approx 1-2 \times 10^{2} \mathrm{~cm}^{-3}$.

2. The clumps identified using the column density PDFs are gravitationally unbound and the external pressure, caused by the turbulent pressure from the diffuse, large-scale cloud surrounding them, can provide significant support for them against dispersal. However, examination of the stability of a small subsample of clumps indicates that they may be overpressurized and either expanding or additionally supported by a component not included in our analysis (e.g. magnetic field support). Then, the physical properties of the clumps resemble those of the clumps often identified from ${ }^{13} \mathrm{CO}$ emission observations as structures of the lowest hierarchical level.

3. In Kainulainen et al. (2009b), we showed that some non-starforming clouds do not show the PDF break, while some of them show a weak break but no gravitationally dominated dense cores. Coupling those results with the physical characteristics of the clumps derived in this paper suggests a picture in which pressure-confined clumps form prior to, or at higher rate compared to, the formation of gravitationally dominated dense cores in the clouds. This suggests that the formation of pressure-confined clumps is a prerequisite for star formation, and introduces a natural threshold for star formation at $A_{\mathrm{V}}^{\text {tail }}$.

4. The star-formation rate in the cloud complexes of our sample correlates strongly with the mass in the structures defined by the $A_{\mathrm{V}}^{\text {tail }}$ threshold, as pointed out recently by Lada et al. (2010), and furthermore, drops abruptly below that surface density (Heiderman et al. 2010). This supports the interpretation laid out in item 3 above. Most importantly, 
the interpretation then provides a physically motivated explanation for the relation between star-formation rate and the amount of dense material in the clouds reported by Heiderman et al. (2010) and Lada et al. (2010).

Acknowledgements. The authors would like to thank Mordecai-Mark Mac Low, Cornelis Dullemond, and Ralf Klessen for enlightening discussions regarding the topic. We would like to thank the anonymous referee for helping us to significantly improve the manuscript. We would also like to thank Jaime Piñeda for providing electronic material for Fig. 3. C.F. has received funding from the European Research Council under the European Community's Seventh Framework Programme (FP7/2007-2013 Grant Agreement No. 247060) for the research presented in this work

\section{References}

Alves, J., Lombardi, M., \& Lada, C. J. 2007, A\&A, 462, L17

André, P., Menśhchikov, A., Bontemps, S., et al. 2010, A\&A, 518, L102

Ballesteros-Paredes, J. 2006, MNRAS, 372, 443

Ballesteros-Paredes, J., \& Mac Low, M.-M. 2002, ApJ, 570, 734

Banerjee, R., Vázquez-Semadeni, E., Hennebelle, P., \& Klessen, R. S. 2009, MNRAS, 398, 1082

Bertoldi, F., \& McKee, C. F. 1992, ApJ, 395, 140

Blitz, L., Fukui, Y., Kawamura, A., et al. 2007, Protostars and Planets V, 81

Bohlin, R. C., Savage, B. D., \& Drake, J. F. 1978, ApJ, 224, 132

Brunt, C. M. 2010, A\&A, 513, A67

Brunt, C. M., Federrath, C., \& Price, D. J. 2010a, MNRAS, 403, 1507

Brunt, C. M., Federrath, C., \& Price, D. J. 2010b, MNRAS, 405, L56

Burrows, D. N., Singh, K. P., Nousek, J. A., et al. 1993, ApJ, 406, 97

Butler, M. J., \& Tan, J. C. 2009, ApJ, 696, 484

Cambrésy, L. 1999, A\&A, 345, 965

Carr, J. S. 1987, ApJ, 323, 170

Caselli, P., Benson, P. J., Myers, P. C., \& Tafalla, M. 2002, ApJ, 572, 238

Cho, W., \& Kim, J. 2011, MNRAS, 410, L8

Crutcher, R. M. 1999, ApJ, 520, 706

Crutcher, R. M., Wandelt, B., Heiles, C., et al. 2010, ApJ, 725, 466

Elmegreen, B. G. 2008, ApJ, 672, 1006

Elmegreen, B. G., \& Falgarone, E. 1996, ApJ, 471, 816

Falgarone, E., Puget, J.-L., \& Perault, M. 1992, A\&A, 257, 715

Federrath, C., Glover, S. C. O., Klessen, R. S., \& Schmidt, W. 2008a, Phys.

Scripta T, 132, 014025

Federrath, C., Klessen, R. S., \& Schmidt, W. 2008b, ApJ, 688, L79

Federrath, C., Klessen, R. S., \& Schmidt, W. 2009, ApJ, 692, 364

Federrath, C., Roman-Duval, J., Klessen, R. S., et al. 2010, A\&A, 512, A81

Froebrich, D., \& Rowles, J. 2010, MNRAS, 406, 1350

Goodman, A. A., Barranco, J. A., Wilner, D. J., \& Heyer, M. H. 1998, ApJ, 504, 223

Goodman, A. A., Pineda, J. E., \& Schnee, S. L. 2009, ApJ, 692, 91

Heiderman, A., Evans, N. J., II, Allen, L. E., et al. 2010, ApJ, 723, 1019
Hennebelle, P., \& Chabrier, G. 2009, ApJ, 702, 1428

Heyer, M. H., Carpenter, J. M., \& Snell, R. L. 2001, ApJ, 551, 852

Kainulainen, J., Lehtinen, K., \& Harju, J. 2006, A\&A, 447, 597

Kainulainen, J., Lada, C. J., Rathborne, J. M., \& Alves, J. F. 2009a, A\&A, 497, 399

Kainulainen, J., Beuther, H., Henning, T., \& Plume, R. 2009b, A\&A, 508, L35, PaperI

Klessen, R. S. 2000, ApJ, 535, 869

Kramer, C., Stutzki, J., Rohrig, R., \& Corneliussen, U. 1998, A\&A, 329, 249

Kritsuk, A. G., Norman, M. L., \& Wagner, R. 2011, ApJ, 727, L20

Krumholz, M. R., \& McKee, C. F. 2005, ApJ, 630, 250

Krumholz, M. R., McKee, C. F., \& Tumlinson, J. 2009, ApJ, 699, 850

Lada, C. J., Muench, A. A., Rathborne, et al. 2008, ApJ, 672, 410

Lada, C. J., Lombardi, M., \& Alves, J. F. 2009, ApJ, 703, 52

Lada, C. J., Lombardi, M., \& Alves, J. F. 2010, ApJ, 724, 687

Larson, R. B. 1981, MNRAS, 194, 809

Lombardi, M. 2005, A\&A, 438, 169

Lombardi, M. 2009, A\&A, 493, 735

Lombardi, M., \& Alves, J. 2001, A\&A, 377, 1023

Lombardi, M., Alves, J., \& Lada, C. J. 2006, A\&A, 454, 781

Lombardi, M., Lada, C. J., \& Alves, J. 2008a, A\&A, 480, 785

Lombardi, M., Lada, C. J., \& Alves, J. 2008b, A\&A, 489, 143

Lombardi, M., Alves, J., \& Lada, C. J. 2010a, A\&A, 519, L7

Lombardi, M., Lada, C. J., \& Alves, J. 2010b, A\&A, 512, A67

Mac Low, M.-M., \& Klessen, R. S. 2004, Rev. Mod. Phys., 76, 125

Maruta, H., Nakamura, F., Nishi, R., et al. 2010, ApJ, 714, 680

McKee, C. F., \& Ostriker, E. C. 2007, ARA\&A, 45, 565

Motte, F., Andre, P., \& Neri, R. 1998, A\&A, 336, 150

Ostriker, E. C., Gammie, C. F., \& Stone, J. M. 1999, ApJ, 513, 259

Ostriker, E. C., Stone, J. M., \& Gammie, C. F. 2001, ApJ, 546, 980

Padoan, P., \& Nordlund, A.. 2002, ApJ, 576, 870

Padoan, P., Nordlund, A., \& Jones, B. J. T. 1997, MNRAS, 288, 145

Pineda, J. E., Rosolowsky, E. W., \& Goodman, A. A. 2009, ApJ, 699, L134

Pineda, J. E., Goodman, A. A., Arce, H. G., et al. 2010a, ApJ, 712, L116

Pineda, J. L., Goldsmith, P. F., Chapman, N., et al. 2010b, ApJ, 721, 686

Ridge, N. A., Di Francesco, J., Kirk, H., et al. 2006a, AJ, 131, 2921

Ridge, N. A., Schnee, S. L., Goodman, A. A., \& Foster, J. B. 2006b, ApJ, 643, 932

Sánchez, N., Alfaro, E. J., \& Pérez, E. 2005, ApJ, 625, 849

Scalo, J., Vazquez-Semadeni, E., Chappell, D., \& Passot, T. 1998, ApJ, 504, 835

Schneider, N., Csengeri, T., Bontemps, S., et al. 2010, A\&A, 520, A49

Skrutskie, M. F., Cutri, R. M., Stiening, R., et al. 2006, AJ, 131, 1163

Solomon, P. M., Rivolo, A. R., Barrett, J., \& Yahil, A. 1987, ApJ, 319, 730

Tassis, K., Christie, D. A., Urban, et al. 2010, MNRAS, 408, 1089

Vázquez-Semadeni, E. 1994, ApJ, 423, 681

Vázquez-Semadeni, E., \& García, N. 2001, ApJ, 557, 727

Vázquez-Semadeni, E., Ballesteros-Paredes, J., \& Klessen, R. S. 2003, ApJ, 585, L131

Ward-Thompson, D., Kirk, J. M., André, P., et al. 2010, A\&A, 518, L92

Williams, J. P., de Geus, E. J., \& Blitz, L. 1994, ApJ, 428, 693

Williams, J. P., Blitz, L., \& Stark, A. A. 1995, ApJ, 451, 252 